Krankenhäuser erhalten seit Einführung der DRGs eine Fallpauschale für die Behandlung eines Patienten, die umso mehr wert ist, je schneller der Patient entlassen wird. Die aktuellen Krankenhausstatistiken weisen deshalb bereits seit geraumer Zeit immer kürzere Liegezeiten, aber eine deutliche Fallzahlzunahme auf. Wenig überraschend ist deshalb, dass die Ausgaben im stationären Sektor erheblich ansteigen.

Die erwähnten Urteile dürften diese Tendenz noch verstärken, denn der niedergelassene Arzt hat praktisch nur eine Chance, sich gegen diesen Teufelskreis zu seinen Lasten zu wehren, wenn er vom Krankenhaus gewünschte prä- oder poststationäre Leistungen konsequent verweigert und an das Krankenhaus zurückdelegiert. Der Leidtragende dieses juristisch ausgelösten Kuriosums ist der Bürger gleich in doppelter Hinsicht: als Patient und als Beitragszahler.

Zumindest die bayrische Krankenhausgesellschaft hat das wohl erkannt. In einer Stellungnahme fordert deren Vorsitzender, dass angesichts der angespannten finanziellen Situation im Gesundheitswesen die Einsparmöglichkeiten an den Schnittstellen zwischen ambulantem und stationärem Sektor besser genutzt werden müssten, indem Doppelvorhaltungen abgebaut, der Informationsfluss verbessert und eine fach- und sektorenübergreifende Behandlung der kurzen Wege organisiert werde. Dabei gehe es nicht um eine Verdrängung des vertragsärztlichen Bereichs, sondern um ein sinnvolles und notwendiges Miteinander.

\title{
Broncholysetest richtig abrechnen
}

— Viele KVen streichen im Rahmen der sachlich-rechnerischen Berichtigung den zweimaligen Ansatz der Ziffer 03330 EBM an jeweils einem Behandlungstag wegen der Untersuchung der Lungenfunktion vor und nach der Broncholyse mit der Begründung, die Ziffer sei nur einmal pro Sitzung abrechenbar. Im Text zur Nr. 03330 EBM ist ein Mehrfachansatz aber nicht ausgeschlossen, und die Systematik des EBM sieht vor, wenn ein mehrfacher Ansatz einer Ziffer des EBM verhindert oder auch ein zeitlicher Ausschluss vorgegeben werden soll, dass dies in der Leistungslegende erwähnt wird. Ist die Vorgehensweise der betreffenden KVen deshalb falsch?

\section{MMW Kommentar}

Kassenärztliche Vereinigungen folgen in Fällen, bei denen eine Auslegung der Gebührenordnung notwendig ist, oft den Ausführungen in Kommentaren. Alle etwas bekannteren Kommentare vertreten im vorliegenden Fall die Auffassung, dass die Nr. 03330 EBM nur einmal je Sitzung berechnet werden kann, auch wenn mehrere Messungen z.B. vor und nach der Applikation bronchospasmolytisch oder bronchokonstriktorisch wirksamer Substanzen erfolgen. Eine nähere Begründung für diese Aussage geben die Autoren der Kommentare allerdings nicht. Da es bisher keine Rechtsprechung zum Thema gibt, ist die Frage der Berechnungsfähigkeit offen und kann unterschiedlich ausgelegt werden.

Bei der Lösung des Problems könnte dabei die Begrifflichkeit bei der Abrechnung im GOÄ-Bereich helfen. Dort kann die Lungenfunktionsprüfung nach den Nrn. 605 und 605 a GOÄ berechnet werden. Beim Broncholysetest kommen die Leistungen nach den Nrn. 605 und 605 a für die notwendige Kontrolluntersuchung jedoch auch dort nur einmal zum Ansatz. Aufschluss darüber findet sich nicht vor-

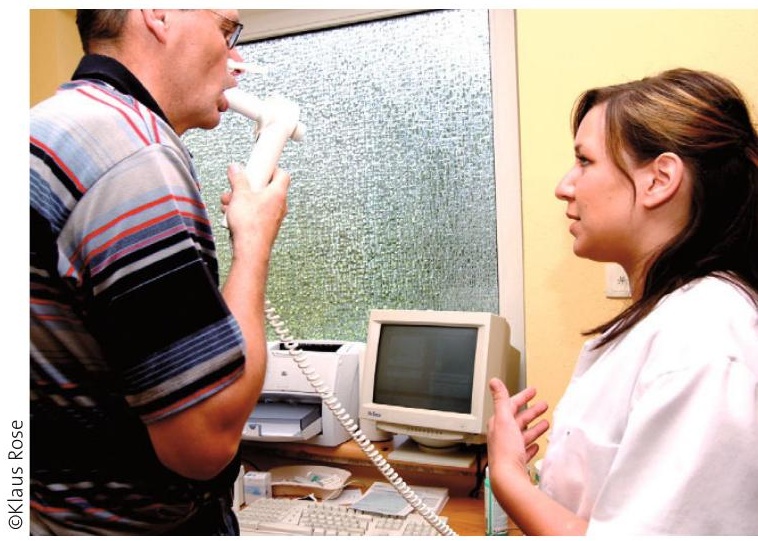

Broncholysetest: zweimal untersuchen, aber nur einmal abrechnen?

dergründig in der GOÄ, sondern vielmehr in der Grammatik und Systematik der deutschen Sprache. Zwar ist die Leistungslegende jeweils im Singular formuliert („Ruhespirografische Untersuchung“ bzw. „Darstellung der Flussvolumenkurve“), doch handelt es sich jeweils um eine Untersuchung. Das Wort „Untersuchung“ als solches wiederum umfasst mehrere einzelne diagnostische Schritte. Dadurch kann in einer Sitzung die spirografische Untersuchung nicht mehrfach berechnet werden.

Die GOÄ bietet jedoch die Möglichkeit, den zusätzlichen Aufwand einer zweiten Funktionsprüfung im Rahmen der Broncholyse entsprechend geltend zu machen. (entweder durch die Nr. 609 GOÄ oder, wenn die Nr. 609 GOÄ nicht zur Abrechnung kommt, durch die Verwendung eines höheren Faktors für die Leistungen nach den Nrn. 605 und 605a).

Diese Möglichkeit ist im EBM nicht vorhanden, sodass zumindest die Forderung nach einer zusätzlichen Berechnung aufrechterhalten werden könnte. Eine juristische Klärung ist dann allerdings nicht zu vermeiden. 